\title{
The Effects of Motif Composition on Impressions of Paintings
}

- In the Case of Katsushika Hokusai’s “Thirty-Six Views of Mount Fuji” -

\author{
Yuka NOJO*, Keiichi MURAMATSU**, Kazuaki KOJIMA*** and Tatsunori MATSUI*** \\ * School of Human Sciences, Waseda University, 2-579-15 Mikajima, Tokorozawa, Saitama 359-1192, Japan \\ ** Graduate School of Human Sciences, Waseda University, 2-579-15 Mikajima, Tokorozawa, Saitama 359-1192, Japan \\ *** Faculty of Human Sciences, Waseda University, 2-579-15 Mikajima, Tokorozawa, Saitama 359-1192, Japan
}

\begin{abstract}
There exist pictures that people have recognized as great, beyond the borders of cultural differences, over the last 100 years. This suggests that such paintings would have certain aesthetic elements in common, and if people are able to recognize them through a shared sense of beauty, it is possible to study what kinds of properties influence people's evaluation of paintings. This study investigated the influence of motif composition on people's impression of Hokusai's paintings. In the experiment, participants were asked to give their impression of the paintings using two kinds of rating scales, the information rate scale (IRS), and the semantic differential (SD) scale. We defined symmetry, complexity and order as the elements of beauty. Results revealed three perceptual features that strongly affect people's impressions of the beauty of paintings.
\end{abstract}

Keywords: Impressions of Paintings, Motif Composition, Semantic Differential

\section{INTRODUCTION}

\subsection{Background and Purpose}

There are pictures which have been almost universally recognized as great pictures for over 100 years, such as Da Vinci's "The Last Supper," and Monet's "Water Lilies." Their existence suggests that there are certain aesthetic elements in common among great paintings, and if people are able to recognize them through a shared sense of beauty, it is possible to identify the properties that influence people's evaluation of paintings.

Many theorists dating back to ancient Greece saw that "one of the factors of beauty is symmetry" $[1,3,7,8,10]$; and symmetry-based order is also the factor [1]. Birkoff, who described the two defining features of beauty, complexity $[4,2,12]$ and order $[3,5]$ as the aesthetic properties, suggested that perceptual features and beauty are closely related.

In this paper, we adopted the "golden ratio" as one of the scales of beauty; this ratio $(1: 1.618)$ exists widely throughout nature and in the arts, such as in the Khufu Pyramid of Egypt (the ratio of the height of its triangular face to half the side of its base), the Venus de Milo (the ratio of its body proportions), the chambered nautilus shell (the ratio of its spiral structure), and so forth. The golden ratio has been introduced into great deal of research that discusses the nature of beauty; for instance, in various art domains, researchers confirmed the existence of the golden ratio by adding auxiliary lines into compositions that used it [15]. In the Oriental art domain, researchers used it for analyzing the structural ratio of Buddha statues [16], while in Kansei engineering, it was adopted to analyze the properties of the attractiveness of a human smile [20].

The psychological model of aesthetic experience judgment [14] showed that affective processing is defined in five steps as "perceptual analyses," "implicit memory integration," "explicit classification," "cognitive mastering," and "evaluation." These affective evaluations are processed sequentially. Perceptual features like "symmetry," "complexity," and "order" are processed in the initial phase. The processing of these perceptual features tends to elicit positive emotions, because of a significant feature they hold, which is an "easy to identify stimuli's physical properties" [18], as indicated by psychological and physiological findings. We followed these traditional theories, and the focus of this experiment was to explore correlations between the perceptual features and the affective state induced by stimuli.

The main issue of this study was to investigate the influence of motif composition on people's impressions of Hokusai's paintings, particularly focusing on their perceptual features. In this analysis, we adopted the perceptual features as "symmetry," "complexity," and "order." As relevant research suggests, "beauty induces pleasurable experience" $[5,9,19,21]$; therefore, we took up "pleasant-unpleasant" as the affective state factor, and "aroused-unaroused" as the influential factor which 
induces a change in feeling. We asked participants to evaluate the original picture and a re-worked version in which the motif composition had been changed, to analyze the relation between perceptual features and the affective impressions of paintings.

If a relationship is revealed, the processes behind human aesthetic experience will be further clarified, and it will be possible to construct "automatic recognition of aesthetic evaluation" which is able to measure the level of beauty underlying systematical algorithms as the final target of our research. First, in focusing on a visual art such as painting, we investigated what elements make paintings beautiful.

\section{ANALYSIS POINT}

We designed the analysis point of this experiment based on the tentative theory that if people experience beauty upon seeing a golden ratio in a motif composition, it will be possible to create "automatic recognition of aesthetic evaluation," a process that can extract the golden ratio of a motif composition to effectively evaluate the level of beauty in paintings.

In focusing on the golden ratio, which is the positional correlation between the outlines of the main motif and others motifs, we propose that a composition that includes the golden ratio is more beautiful than a composition that does not.

The hypothesis was proposed according to findings in two previous studies; first, the perceptual features of a painting depend on people's perceptions of a motif's outline [22], and second, some pictures evaluated as beautiful tend to have some kind of positional rule, such as the golden ratio [15].

According to recent research on visual information, there are orientations of selective cells in our brain that respond only to a specific angle, and if people were to lose this function, it would be impossible for them to recognize lines and objects [22]. Visual pattern information is processed in the brain hierarchically within the visual cortex, and line information is integrated into images as an initial transfer step [15], which suggests that outlines have a vital role in pattern information [13], especially in art. Moreover, the brain captures the essential features of objects or situations from visual images [22], and it has been suggested that vision is an "active procedure extracting necessary information to classify objects" [22]. Thus, it may be that people recognize motifs in paintings from their outlines, by judging the perceptual features of "symmetry," "complexity," and "order" from among various visual features.

The golden ratio, as mentioned, has been widely used in previous research as a criterion of measuring the level of beauty in paintings; thus, it is possible to see this as one criterion in assessing how beautiful paintings are.

\section{EXPERIMENT}

\subsection{Experimental Design}

As the target of this paper was to clarify how the perceptual features of a painting affected viewer impression, we showed two images with different perceptual features, and performed an evaluation of the impressions. In the painting, we used a motif that comprised a golden ratio, and then changed the perceptual features of the entire composition. As a result, the perceptual features between the original painting and the re-worked one were different, even though both contained similar motifs. By comparing the impression viewers received from each painting, it was possible to study the effects of perceptual features on people's impressions.

According to previous research [6], the background information of a picture has a strong influence on people's impressions of paintings, so we did not disclose any information about the stimuli to subjects before the test. However, there was still the possibility that Hokusai's pictures were familiar or could be considered "great pictures," and subjects may have been able to recognize the main motifs of the stimuli, and thus bias the results.

\section{MATERIALS}

Six of Hokusai's paintings from the series "Thirty-Six Views of Mount Fuji" were selected. The reasons we selected these were that (1) they are "great pictures" renowned for more than a century both domestically and overseas, (2) they are line-drawn pictures in which each motif can be easily recognized, and (3) each motif's figure and positional proportion can be easily compared and analyzed digitally.

As referred to in the title "Mount Fuji" this series shows the subject explicitly. Even though we did not disclose the title to participants in advance, it was still possible to easily predict what the main motif was, from either name recognition or participants' memory of the picture. To avoid preexisting bias, we avoided pictures in which (1) the size of the motif of Mount Fuji was extremely large, and (2) it is difficult to avoid the influence of color contrast after gray-scale processing. We prepared twelve pictures in total, including six original and six re-worked pieces. 
In this experiment, we saw the golden ratio as "a visual balance which is created by dividing an area" [15], with two underlying rules: (1) the ratio of the lesser segment to the greater segment should be $1: 1.618$, and (2) the dividing point of either the greater or lesser segment should be on the center line of the Mount Fuji motif. Figure 1 shows the original picture on the left side, which has the golden ratio indicated by a solid arrow and a dashed arrow, and the re-worked picture on the right side in which the Mount Fuji motif was moved to disrupt the ratio.
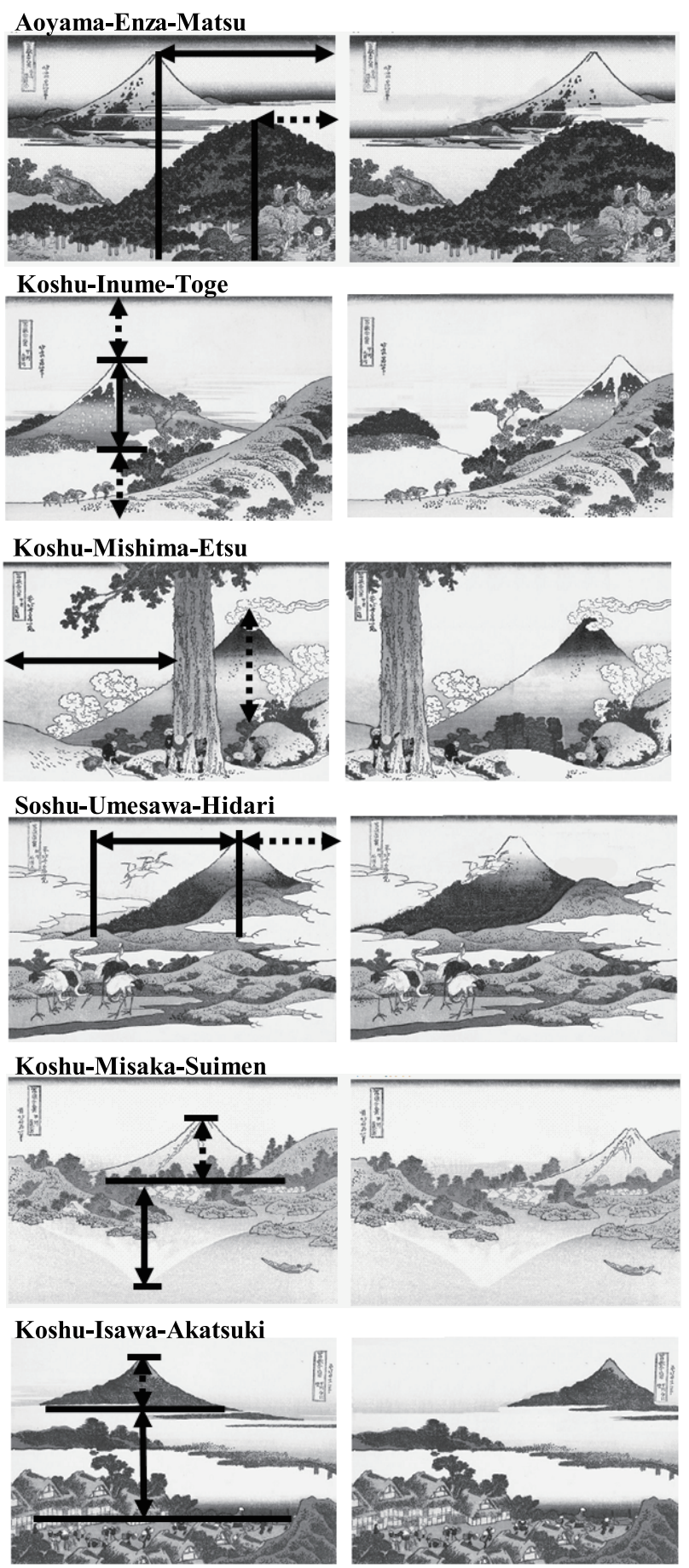

Figure 1: Six pairs of stimuli.

Originals on the left, re-worked on the right.
In "Aoyama-Enza-Matsu," the golden ratio is the distance from the right edge to the center line of Mount Fuji, and then to the center of the mound; therefore, in the re-worked picture, we moved the Mount Fuji motif to the right side to disrupt this ratio. "Koshu-Inume-Toge" shows the golden ratio reflected in the height of Mount Fuji and the width of the spatial area above and below Mount Fuji; to break the ratio, we moved Mount Fuji to the back of the right side mound. In "Koshu-Mishima-Etsu," the ratio is found in the spaces to the left and right of the tree and the height of Mount Fuji; we disrupted it by moving the tree farther to the left. This stimulus also has another golden ratio in the distance from the foot of Mount Fuji and its height. "Soshu-Umesawa-Hidari" shows the ratio in the distance from the beginning of the slope of the mountain to its top, and the space between the top and the right edge of the picture; we moved Mount Fuji to the center to disrupt the ratio. In "Koshu-Misaka-Suimen," the ratio is found in the height of Mount Fuji and the height of Fuji's reflection on the lake; we moved the Fuji motif farther to the right to disrupt the ratio. "KoshuIsawa-Akatsuki" shows the ratio in the height of Mount Fuji and the distance from foot of the mountain to the village road; we moved Fuji again to the right side to disrupt the ratio.

\section{METHOD}

We employed the Semantic Differential (SD) method, which is a widely used instrument in psychology and other fields, to measure people's impressions of the pictures. The one we adapted for this study consisted of a set of 20 bipolar adjective pairs that are each rated along a 7-point scale. In addition, we chose the items from a previous study in which 14 adjective pairs [11], translated into Japanese (dense-sparse, asymmetrical-symmetrical, usual-surprising, crowded-uncrowded, simple-complex, immediate-distant, heterogeneous-homogeneous, similarcontrasting, novel-familiar, patterned-random, intermittentcontinuous, common-rare, small scale-large scale, varied-redundant), were chosen from the Information Rate Scale (IRS; [17]). The IRS can eliminate influence from the participant's mood, and is an effective measure in evaluating environmental information. As beauty is considered to be related to emotions, six pairs of the SD method (aroused-unaroused, satisfied-unsatisfied, wide awake-sleepy, pleased-annoyed, exited-calm, happyunhappy; [11]) were adapted. The participants, six students (one female and five males) aged 20 and 21 years old, viewed the stimuli presented on a computer 
screen, without a time limit. They were asked to express their preference by selecting which adjective best described the feeling they received from the painting.

\section{RESULTS}

A maximum likelihood factor analysis using varimax rotation was performed for the evaluation scores of the experiment. We repeated this process without three of the factors whose communality scores were particularly low. Finally, six factors (cumulative contribution ratio $56.35 \%$ ) were extracted. Table 1 shows the factor loadings of each scale item after varimax rotation.
Referring to previous research [11], we defined six factors that showed factor loadings of less than 0.40; the first factor was defined as Originality, the second, Density, the third, Influential, the fourth, Continuity, the fifth, Arousal, and the sixth, Similarity. Originality, Density, Continuity, and Similarity all contained only IRS factors, while Influential contained only SD factors; Arousal included both scale factors. There was no particular problem in the analysis.

Table 2 shows a comparison of the loading factors of people's impressions of the original and re-worked paintings, and Table 3 shows the results of a validation of our hypothesis.

Table 1: The factor loadings of each scale item after varimax rotation.

\begin{tabular}{|c|c|c|c|c|c|c|c|}
\hline & Originality & Density & Influential & Continuity & Arousal & Similarity & Communality \\
\hline common-rare & .7389 & .0807 & .0841 & -.1099 & -.0393 & -.0068 & .5732 \\
\hline small scale-large scale & .6811 & .1087 & .0795 & -.0183 & -.0919 & -.1409 & .5107 \\
\hline usual-surprising & .6741 & .1815 & -.1904 & -.1586 & .1238 & -.0066 & .5641 \\
\hline heterogeneous-homogeneous & .6132 & .2730 & -.0616 & .0341 & -.0141 & .1733 & .4856 \\
\hline novel-familiar & .4738 & .0319 & .1192 & -.1371 & .0223 & .2363 & .3148 \\
\hline crowded-uncrowded & .1392 & .8747 & .0670 & -.0105 & -.0112 & .0845 & .7963 \\
\hline dense-sparse & .1091 & .7668 & -.0845 & -.0139 & -.0008 & -.0211 & 6077 \\
\hline simple-complex & .2815 & .4910 & .1756 & -.1063 & -.0169 & .1536 & .3864 \\
\hline pleased-annoyed & .0729 & .1056 & .8115 & .1115 & .0544 & -.0306 & .6912 \\
\hline aroused-unaroused & -.4760 & -.0165 & .6219 & -.0799 & .1938 & -.0140 & .6578 \\
\hline satisfied-unsatisfied & .0351 & -.1053 & .4852 & .0144 & .6217 & -.0107 & .6346 \\
\hline happy-unhappy & .2506 & .0054 & .3551 & -.0156 & .2404 & -.0410 & .2486 \\
\hline intermittent-continuous & .0017 & -.0176 & -.0582 & .9253 & .0641 & -.0788 & .8703 \\
\hline patterned-random & -.2121 & -.0525 & .1116 & .6174 & -.0260 & .0538 & .4450 \\
\hline wide awake-sleepy & .0549 & .1450 & .0882 & -.0918 & .6121 & .1172 & .4287 \\
\hline varied-redundant & .2208 & .1873 & -.0179 & -.2214 & -.4851 & .0487 & .3709 \\
\hline similar-contracting & .0598 & .1381 & -.0855 & -.0001 & .0778 & .9793 & .9950 \\
\hline Square sum of factor loading & 2.57142 & 1.82176 & 1.54811 & 1.38329 & 1.13216 & 1.12410 & 9.58084 \\
\hline Factor contribution & 15.12600 & 10.71624 & 9.10651 & 8.13700 & 6.65977 & 6.61236 & 56.35788 \\
\hline cumulative contribution ratio & 15.12600 & 25.84224 & 34.94875 & 43.08575 & 49.74552 & 56.35788 & \\
\hline
\end{tabular}

Table 2: The comparison table of factor loadings.

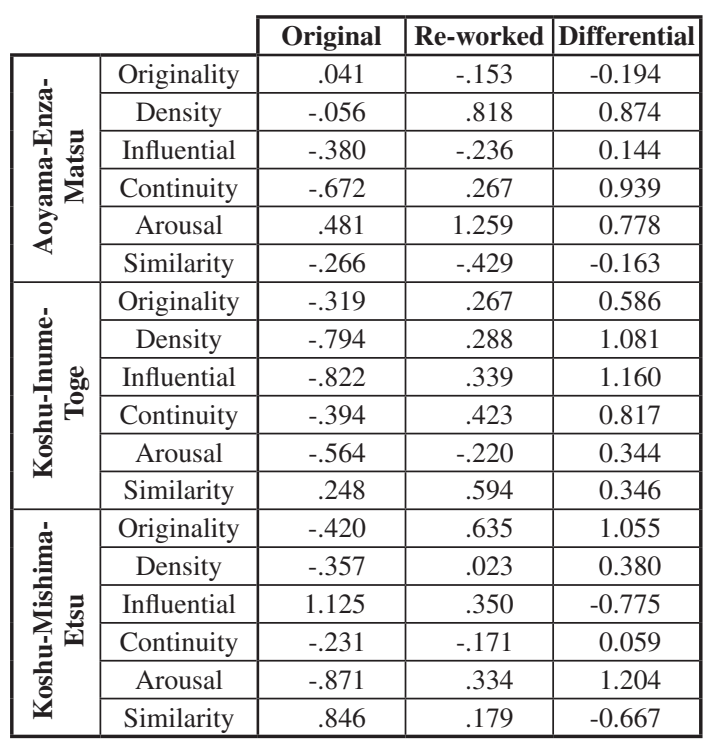

\begin{tabular}{|c|c|c|c|c|}
\hline & Original & Re-worked & Differential \\
\hline \multirow{6}{*}{ 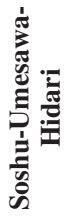 } & Originality & .638 & -.058 & -0.696 \\
\hline & Density & .037 & -.390 & -0.428 \\
\hline & Influential & .108 & .515 & 0.407 \\
\hline & Continuity & -.832 & .226 & 1.059 \\
\hline & Arousal & -.358 & -.547 & -0.189 \\
\hline & Similarity & -.107 & -.859 & -0.751 \\
\hline \multirow{6}{*}{ 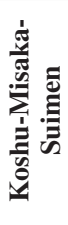 } & Originality & .413 & -.582 & -0.995 \\
\hline & Density & .113 & .144 & 0.031 \\
\hline & Influential & .763 & -.382 & -1.144 \\
\hline & Continuity & -.369 & .090 & 0.459 \\
\hline & Arousal & -.372 & .380 & 0.752 \\
\hline & Similarity & .198 & .095 & -0.104 \\
\hline \multirow{6}{*}{ 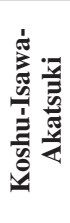 } & Originality & -1.086 & .624 & 1.710 \\
\hline & Density & -.295 & .665 & 0.960 \\
\hline & Influential & -.521 & -.490 & 0.031 \\
\hline & Continuity & 1.213 & .004 & -1.209 \\
\hline & Arousal & -.194 & -.059 & 0.135 \\
\hline & Similarity & -.237 & -.255 & -0.018 \\
\hline
\end{tabular}


Table 3: The results of hypothesis validation. $\mathrm{S}=$ supported, $\mathrm{R}=$ rejected

\begin{tabular}{|l|c|c|c|c|c|}
\hline & Symmetry & Complexity & Order & Pleasantmess & Arousal \\
\hline Aoyama-Enza-Matsu & & R & S & & R \\
\hline Koshu-Inume-Toge & R & R & S & R & R \\
\hline Koshu-Mishima-Etsu & S & & & S & R \\
\hline Soshu-Umesawa-Hidari & S & S & S & R & \\
\hline Koshu-Misaka-Suimen & & & S & S & R \\
\hline Koshu-Isawa-Akatsuki & & R & R & & \\
\hline
\end{tabular}

\subsection{Results of the Validation of Perceptual Features and Hypothesis}

In this subsection, we validated the hypothesis for each perceptual feature-csymmetry," "complexity," "order," "pleasantness," and "arousal"-based on the result of a factor analysis.

"Symmetry"

The Similarity factor, which included the "asymmetricalsymmetrical" adjective pair factors and expressed "symmetry," was based on the hypothesis that a lower score gives a more symmetric impression. The result showed that the scores were low in four cases, and significantly low in two cases. With regard to our hypothesis that greater "symmetry" equals greater beauty, the result showed that the golden ratio had low influence for this factor, that means and the hypothesis was not supported.

\section{"Complexity"}

The Density factor, which included the "simplecomplex" adjective pair factors, reflected "complexity" in that a lower score meant a less complex impression. With reference to the definition of "aesthetic measure" [3], we analyzed the result based on the hypothesis that lower "complexity" meant more "beautiful." In five cases, the re-worked stimuli's factor loading changed to positive, meaning that the re-worked piece was more beautiful than the original; therefore, the golden ratio had low influence for this factor, and the hypothesis was not supported.

\section{"Order"}

The Continuity factor, which included the "patternedrandom" adjective pair factors, reflected "order" in that a lower score meant a stronger impression of "order." With reference to the definition of "aesthetic measure" [3], we analyzed the result based on the hypothesis that a higher "order" score meant that the picture was more beautiful. In five cases, the re-worked stimuli's factor loading changed to positive, meaning that the re-worked paintings were not as beautiful as the original; therefore, the golden ratio had a strong impression for this perceptual feature and the hypothesis was supported.

\section{"Pleasantness"}

For the "Influential" factor, which included the "pleased-annoyed" adjective pair factors and reflected the "pleasant" impression, we analyzed the result based on the hypothesis that a higher "pleasant" score meant subjects thought the picture was more beautiful. In four cases, the re-worked piece's loading factors increased, and in two cases decreased; therefore, the golden ratio had an ambiguous influence in this factor, and the hypothesis was not supported.

\section{"Arousal"}

In the Arousal factor, which included the "awakesleepy" adjective pair factors, we interpreted that a higher "awake" score meant a greater impression of "arousal". We analyzed the result based on the hypothesis that a higher "arousal" score meant that subjects thought a picture was more beautiful. In five cases, the loading factors increased, therefore the golden ratio had strong influence in this factor, and the hypothesis was supported.

\section{DISCUSSION}

\subsection{The Golden Ratio and the Beauty of Paintings}

The results showed that the hypothesis of this experiment - that a composition including the golden ratio is more beautiful than one that does not include it-was supported in 9 cases out of 24 , but not supported in 11 cases.

The perceptual feature "order" strongly supported the hypothesis in four cases, while "arousal" did not support it in four cases. The three other perceptual features"symmetry, "complexity," and "pleasantness"- supported the hypothesis half of the time.

Therefore, it was found that the golden ratio influenced both "order" and "arousal," but on the other hand, it barely influenced the "symmetry," "complexity" and "pleasantness" factors. Moreover, the influence of the golden ratio on people's impression of the beauty of paintings remains unclear, but it can be said to be one factor that comprises people's overall impressions of paintings.

\subsection{The Perceptual Features and Beauty of Paintings}

Using the five perceptual features defined in this experiment, "symmetry," "complexity," "order," "pleasantness," and "arousal," we analyzed the difference of people's impressions of the original and re-worked paintings, and what kind of features were most strongly linked to impression.

There are three cases that supported the hypothesis with more than two perceptual features: "Koshu-Mishima-Etsu," 
"Soshu-Umesawa-Hidari," and "Koshu-Misaka-Suimen." The particular points of these cases are the large motif size, how easy it is to recognize the features of the motif, and how easily the main motif can be compared with other motifs; with the features present in these pictures, there is a strong impression of beauty. To be more precise, in "Koshu-Mishima-Etsu," the motif forms are obviously very unique, with the triangle of Mount Fuji and the quadrangular tree trunk; in such a picture, it is easy to compare each motif's size, positional relation and size. In "Soshu-Umesawa-Hidari," both the main motif of Mount Fuji and lesser motif of the small mound are easy to recognize for their size and clear contrast between them. In "Koshu-Misaka-Suimen," Mount Fuji and its reflection on the lake show a mirrored symmetry, giving people who view it a great impression of "symmetry." If, due to a disruption of this "symmetry," "pleasantness" is lowered, it can be said that "symmetry" has a stronger influence on "pleasantness" than the golden ratio.

Regarding "complexity," in three cases that rejected the hypothesis, the results showed that the re-worked pictures were more beautiful, specifically those whose motifs were moved to the right side. From this point, there are possibilities that "complexity" was affected not by the golden ratio, but rather by "symmetry"; thus, "symmetry" would again have stronger influence than "complexity" on people's impressions of paintings.

"Order" gave strong support to the hypothesis for three cases out of four, especially after moving Mount Fuji to the right side, so that it overlapped with the smaller mounds. From this, the fluctuation of motif overlap and composition shows the obvious influence "order" on people's impression of beauty.

In this experiment, especially within the five perceptual features, the results showed that "symmetry," "complexity," and "order" had strong influences on participants' impressions of a painting's beauty. The word "order," which is one of the components of Birkoff's Aesthetic Measure formula, includes in its definition the word "sequence." As both words have almost the same nuances, it is possible to change "order" to "sequence."

According to the psychological model of aesthetic experience judgment [18], precisely "symmetry," "complexity," and "sequence" are affectively evaluated at the initial phase of processing, and have the feature of making it "easy to identify stimuli's physical properties." As positive feeling is equal to feelings of pleasure, and a pleasurable feeling is one factor that leads us to judge something as beautiful, the perceptual features of "symmetry," "complexity," and "order" have thus a strong relationship with beauty, and thus allow us to easily sense the beauty in paintings.

Moreover, the results show that the three properties of motifs that most influence our impression are (1) motif size, (2) the ease of recognition of motif composition, and (3) the balance of motif composition.

\section{CONCLUSION}

For the final target of our research, "automatic recognition of aesthetic evaluation," from the result of this experiment we found three perceptual features that influence our impressions of a painting's beauty.

However, as in the case of "Koshu-Inume-Toge," there remain some questions - in the first place, do participants sense the existence of the golden ratio from motif composition? In recognizing that the ratio had been broken, did their impressions change as a result? Alternatively, was their change in impression not related to the ratio? Did their impressions change due to a change in the symmetry of motif composition? How did they perceive the edge lines of each motif, and is this a result of recognizing them? To find answers to these questions, there is a need for further research on the influence of paintings' motifs, including addressing concerns such as how to select appropriate measures and motif forms to find appropriate criteria for humanity's sense of beauty.

Recently, the relationship between the golden ratio and fractals [15] has been pointed out. It is said that beauty existing in both fractal and nature forms are based on the golden ratio. In the current experiment, the results showed that the effects of the golden ratio on people's impressions of beauty is low; however, in further research, we would like to study the measurement of beauty by including fractals, which has a high availability ratio not only within paintings but also in nature.

\section{ACKNOWLEDGEMENTS}

This work was partially supported by a Grant-in-Aid for Challenging Exploratory Research (21650047).

\section{REFERENCES}

1. Arnheim, R. (1974). Art and visual perception: a psychology of the creative eye, University of California Press, San Francisco, CA, USA.

2. Berlyne, D. E. (1970). Novelty, complexity and hedonic value, Perception and Psychophysics, 8(5), pp.279-286. 
3. Birkoff, G. D. (1933). Aesthetic Measure, Harvard University Press, Cambridge, MA,USA, pp3-15.

4. Eisenman, R. (1967). Complexity-simplicity: 1.Preference for symmetry and rejection of complexity, Psychonomic Science, 8(4), pp.169-170.

5. Feagin, S. L. (1996). Reading with feeling, the aesthetics of appreciation, Cornell University Press, New York, USA.

6. Franklin, M. B., Becklen, R. C., and Doyle, C. L. (1993). The Influence of titles on how paintings are seen, Leonardo, 26(2), pp.103-108.

7. Gombrich, E. H. (1984). The sense of order, a study in the psychology of decorative art, Phaidon Press, Oxford, Great Britain.

8. Gombrich, E. H. (1995). The story of art, Phaidon Press, Oxford, Great Britain.

9. Herzberger, B., Rentschler, I., and Epstein, D. (1989). Beauty and the Brain, Biological Aspects of Aesthetics, Birkhauser Basel Boston Press, Boston, USA.

10. Humphery, D. (1997). Preferences in symmetries in drawings: Asymmetries between ages and sexes, Empirical studies of arts, 5(1), pp.41-60.

11. Ishibashi, Y., and Takahashi, S. (2006). The influence of information about drawing technique on impression of paintings, Japanese Journal of Psychology, 77(2), pp.124-131.

12. Jacobsen, T., and Hofel, L. (2002). Aesthetic Judgments of novel graphic patterns: analysis of individual judgments, Perceptual and Motor Skills, 95(3), pp.755-766.

13. Kikuchi, M., and Oguni, S. (2005). Relation between Contour Integration and Figure-Ground Perception, The institute of electronics, information and communication engineers, 104(760), pp59-62.

14. Leder, H., Belke, B., Oeberst, A., and Augustin, D. (2004). A Model of Aesthetic Appreciation and Aesthetic Judgments, British Journal of Psychology, 95(4), pp.489-508.

15. Livio, M. (2003). The Golden Ratio: The Story of PHI, the World's Most Astonishing Number, Broadway Books, New York, USA.

16. Matsubara, T. (2008). Analysis of Horyuji Mural Painting from the Golden Ratio of body proportion of Bosatsu statue, Buddhism art, The Mainichi Newspapers Press., Tokyo, Japan, 296, pp.9-29.

17. Merabian, A., and Russell, J. A. (1974). An approach to environmental psychology, MIT Press, London, Great Britain, pp.77-94

18. Reber, R., Schwarz, N., and Winkielman, P. (2004). Processing Fluency and Aesthetic Pleasure: Is Beauty in the Perceiver's Processing Experience?, Personality and Social Psychology Review, 8(4), pp.364-382.

19. Santayana, G. (1995, Original work published in 1896). The sense of beauty, Dover Publications, New York, USA, pp.11-31.

20. Sugawara, T., Yamada, N., Sadoyama, T., Hosoya, S., and Igushi, T. (2004). Geometrical Features of the Attractive Smile, Collection of papers of Kansei engineering and emotion research, 5(1), pp.53-58.

21. Tatarkiewicz, W, edited by Harrell, J. (1970). History of aesthetics vol.1,2,3, Polish Scientific Publishers, Warszawa, Poland.

22. Zeki, S. (2000). Inner vision: An exploration of art and the brain, Oxford University Press, New York, USA.

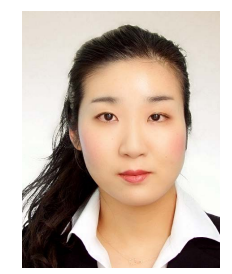

Yuka NOJO

She is a student of Department of Human Informatics and Cognitive Sciences, School of Human Sciences, Waseda University, Japan. She's been working on consilience in human sense of beauty. The main focus of her research is the Kansei evaluation for understanding the knowledge acquisition mechanism. She has been a member of Japan Society of Kansei Engineering and The Japanese Society for Artificial Intelligence since 2012.

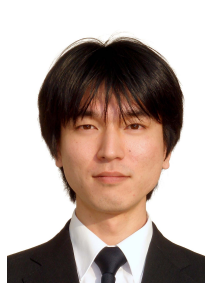

\section{Keiichi MURAMATSU}

Keiichi Muramatsu is a research associate of Faculty of Human Sciences, Waseda University since 2012. He received B.A. (2007) and M.A. (2009) in Human Sciences from Waseda University. He was a Japan Society for the Promotion of Science (JSPS) Research Fellow (DC2) in 2010-2012. His current research interests are ontological descriptions on structure of human mind in the research fields of aesthetics, color science and learning sciences.

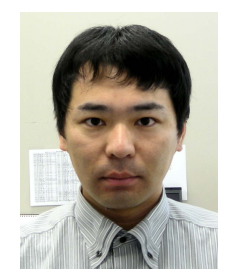

Kazuaki KOJIMA

Kazuaki Kojima is an Assistant Professor in Faculty of Human Sciences, Waseda University, Japan. He received Ph. D. in Human Science from Nagoya University in 2007. He is engaged in research of Artificial Intelligence, Cognitive Science and Educational Engineering. His research interests are in human and computational creativity. From 2007 to 2009, he was a Research Associate in Faculty of Human Sciences, Waseda University. In 2012, he won the best paper award in Japanese Society in Information and Systems in Education.

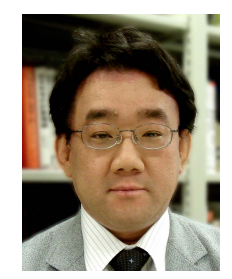

Tatsunori MATSUI

Tatsunori Matsui is a Professor of Knowledge Science and Artificial Intelligence Lab. at Faculty of Human Sciences, Waseda University, Tokyo Japan since 2004, after contributed for 7 years as an Associate Professor to Graduate School of Information Systems, the University of Electro-Communications, Tokyo Japan. Currently, his research interests are Kansei Information Science, Artificial Intelligence in Education, Mathematical Analysis for Educational Data, Statistical Science and so on. He is a member of IEEE, ACM, IEICE, IPSJ, JSAI, JSiSE and so on. 